\title{
Rhodomonas PE545 fluorescence is increased by glycerol
}

\author{
Chanoknard Karnjanapak ${ }^{\mathrm{a}}$, I-Shuo Huang ${ }^{\mathrm{b}}$, Preyanut Jaroensuk ${ }^{\mathrm{a}}$, Sutaporn Bunyajetpong ${ }^{\mathrm{a}, *}$, \\ Paul V. Zimba ${ }^{\mathrm{c}}$, F. Gerald Plumley ${ }^{\mathrm{a}, \mathrm{d}}$ \\ a Department of Marine Science, Faculty of Science, Chulalongkorn University, Bangkok 10330 Thailand \\ b Virginia Institute of Marine Science, Gloucester Point, Virginia 23062 USA \\ c Center for Coastal Studies, Texas A\&M University Corpus Christi, Corpus Christi, Texas 78412 USA \\ ${ }^{d}$ Aquatic Resources Research Institute, Chulalongkorn University, Bangkok 10330 Thailand
}

*Corresponding author, e-mail: sutaporn.b@chula.ac.th

Received 5 Dec 2020

Accepted 27 May 2021

\begin{abstract}
Phycobilins are photosynthetic pigments found in three ecologically important groups of algae: cyanobacteria, red algae, and cryptophytes. These compounds are covalently attached to proteins, which can be technically difficult to analyze compared with other photosynthetic pigments (e.g., chlorophylls and carotenoids). In this study, glycerol was demonstrated to uncouple PE545 (phycoerythrin 545), the phycobilin complex of Rhodomonas spp., from its role as a light-harvesting pigment: the fluorescence signal was increased 15-34 times, and ETR (electron transport rate) was no longer light-dependent at high light intensities. Glycerol induced fluorescence provided a simple and inexpensive protocol to ascertain the pool size of autofluorescent PE545 as well as total PE545 cell content in Rhodomonas. 20-30\% glycerol was optimal for monitoring increased PE545 fluorescence with a stable signal for at least $1 \mathrm{~h}$ after glycerol addition. A substantial portion of PE545 passed through different-sized filter pads in cells with added glycerol, which suggests that glycerol may facilitate extraction of this pigment-protein complex from $R$. salina, a process that can be difficult under certain growth conditions. However, at high concentrations and/or after extended periods of contact, glycerol reduced recovery of PE545, suggesting destruction of the compound. In another approach, we found that PE545 can be recovered from acetone-extracted cells using low concentrations of SDS (sodium dodecyl sulfate) in low $\mathrm{pH}$ buffers. The finding provides samples for analyses of both chlorophylls and carotenoids in acetone and PE545 from the cell pellet. Rhodomonas spp. plays an important role in nature; hence methods presented here should help determine its natural abundance and distribution.
\end{abstract}

KEYWORDS: cryptophyte, Rhodomonas salina, pigment, phycoerythrin, chlorophyll

\section{INTRODUCTION}

Phycobilins play a critical role as photosynthetic light-harvesting antenna in red algae, cyanobacteria, glaucophytes, and cryptophytes. They facilitate utilization of spectral regions that, otherwise, are absorbed poorly by chlorophylls (Chl) and carotenoids. Phycobilin-containing algae and cyanobacteria are important members of the phytoplankton and/or the benthic communities in many freshwater, brackish, and marine habitats. The phycobilin structure is an open-chain tetrapyrrole chromophore covalently attached to apoproteins, collectively termed a phycobiliprotein. The covalent attachment of bilins to proteins conveys a unique biophysical characteristics that alters the biochemistry in such a way that they are challenging to extract and analyze $[1,2]$. Phycobilins lack pho- tochemical activity, but funnel excitation energy to photosynthetic reaction centers (either PSI or PSII). Phycobilin autofluorescence refers to the emission of light energy as fluorescence, rather than the normal/typical transfer of energy to photosynthetic reaction centers [3]. A few species exhibit high phycobilin autofluorescence, indicating that at least some of the pigments are not connected to the photosynthetic apparatus. In situ phycobilin autofluorescence was part of the rational that led to the use of glycerol to completely uncouple phycobilins in Synechococcus spp. [3] and the development of a strategy for use of glycerol to identify, quantitatively and qualitatively, the abundance of this ubiquitous and ecologically important cyanobacterial species in natural samples [4]. Rhodomonas spp., a cryptophyte, also has high phycobilin autofluorescence from PE545, the red colored antenna pigment (phy- 
coerythrin, PE). A preliminary study [5] provides evidence that glycerol increases PE545 fluorescence in $R$. salina. The data presented here support and extend these initial observations.

Methods for analysis of cryptophyte phycobilins, such as the red-colored PE545 lightharvesting complex of Rhodomonas spp., are either time-consuming, preparation intensive, or both. It is useful to separate phycobilin methods into two steps, extraction and analysis. The first step, extraction, can be accomplished in some Rhodomonas isolates with ease and very high efficiency [2], while extraction in other strains requires elevated temperatures [6] or detergents $[1,5]$. Even with complete or nearly complete extraction, the second step, analysis, can be difficult and/or not adequately described in experimental methods. The quantification, an important component of analysis, routinely involves spectrophotometric measurement of absorbance, which can be difficult when samples contain contaminants with overlapping absorption bands (e.g., Chl-protein complexes). Zimba [1] discussed the inherent issues and problems associated with the seemingly simple and time-honored spectrophotometric analysis of phycobilins. In other words, time-intensive steps [7] may be unavoidable, which limits experimental work to only a handful of samples and virtually eliminates field studies, such as ship-board based work. The simple, rapid, and inexpensive means of phycoerythrin analyses in Synechococcus spp. using glycerol is a welcome addition to the arsenal for these cyanobacteria $[3,4]$. The current study extends this glycerol-based technique to a cryptophyte, Rhodomonas spp.

Rhodomonas spp. is arguably the most important and/or most commonly observed cryptophyte in nature, and it also grows well in laboratory cultures. Rhodomonas spp. thrives in turbid and low light environments due to its highly efficient green light harvesting phycobiliproteins and unique dual light-harvesting systems; and/or because it relies upon a variety and flexibility of trophic strategies (i.e., photo-, mixo-, hetero-, and phagotrophy) for carbon and energy assimilation. Cryptophytes are considered the preferred or optimal prey for numerous protist and mesozooplankton grazers [8]. The heavy grazing pressure may lead to low cell numbers of cryptophytes in nature, which belies the importance of these algae in the ecosystem food web. Despite heavy grazing pressures, cryptophytes can occur at high densities in nature under certain conditions [9-11].

In this report, we provided details of techniques that permitted simple and reliable qualitative assessment of phycobiliproteins from a common cryptophyte, Rhodomonas spp. The glycerol technique is similar to a technique used with Synechococcus spp., an ecologically important cyanobacterium [3, 4], and Prochlorococcus marinus [12]. The technique used for Rhodomonas spp. in this study relied on glycerol to uncouple phycobiliproteins from transferring excitation energy to the PSI and/or PSII reaction centers, with formation of unique identifiable fluorescence emissions from the phycobilins. The use of glycerol to identify Synechococcus spp. in coastal and estuarine habitats has become widespread $[13,14]$. Since the seminal work with glycerol-induced fluorescence in Synechoccocus spp. $[3,4]$, another cyanobacterium $P$. marinus $[12]$ and now Rhodomonas have been shown to share this glycerol-dependent phenotype, making it potentially more difficult to correctly interpret results in ecosystems where these species co-exist. The results and implications of this technique with Rhodomonas spp. were also reported in the present study.

\section{MATERIALS AND METHODS}

\section{Algal cultures}

Two Rhodomonas cultures were maintained at either the Chulalongkorn University (CU), Bangkok, Thailand, or the Center for Coastal Studies (CCS), Texas A\&M University Corpus Christi, Texas, USA. Rhodomonas salina, kindly provided by Saskia Ohse and Dr. Alexandra Kraberg (Alfred Wegener Institute, Helgoland, Germany), was grown at $\mathrm{CU}$ in Daigo IMK medium $(\mathrm{pH}$ 8) made from commercially available pre-mix packages (Wako Pure Chemical Industries, Ltd., Japan) using filtered seawater (GF/C filter paper, $1.2 \mu \mathrm{m}$ porosity) at $28 \mathrm{ppt}$ prior to autoclave sterilization. Cultures were maintained at $25^{\circ} \mathrm{C}$ under a photon flux of $54 \mu \mathrm{mol} \mathrm{m}^{-2} \mathrm{~s}^{-1}$ using cool white fluorescent illumination with light:dark cycles of 12:12 h. The second Rhodomonas strain used in this study, Flour Bluff Texas (R-FBT), was isolated from Nueces Bay, Texas and was grown at CCS. Cultures were maintained at $20^{\circ} \mathrm{C}$ in $\mathrm{f} / 2$ media at $32 \mathrm{ppt}$ under a photon flux of $30 \mu \mathrm{mol} \mathrm{m}{ }^{-2} \mathrm{~s}^{-1}$ with light:dark cycles of 12:12 h. For the first strain, small aliquots $(\sim 1 \mathrm{ml})$ of rapidly growing R. salina culture were transferred to $250 \mathrm{ml}$ Erlenmeyer flasks containing $125 \mathrm{ml}$ of fresh culture medium; cells in early exponential phase growth (i.e., approximately 2 days after inoculation) were used for analysis. Some data reported here were extracted from an MSc thesis [5]; for these experi- 
Table 1 Increases of glycerol-induced fluorescence of PE545 in R. salina under different growth regimes and/or with different glycerol concentrations.

\begin{tabular}{|c|c|c|c|c|}
\hline Glycerol concentration & Media $^{a}$ & Culture age $\mathrm{e}^{\mathrm{b}}$ & F585 increase ${ }^{c}$ & Reference \\
\hline 20 or $30 \%$ glycerol & D-IMK & $\mathrm{EE}$ & $25-33$ & This study \\
\hline 20 or $30 \%$ glycerol & D-IMK & LS & $15-19$ & This study \\
\hline $20 \%$ glycerol & D-IMK & LLS & $24-34$ & This study \\
\hline $50 \%$ glycerol & $\mathrm{f} / 2$ & ES & 11 & {$[5]$} \\
\hline $50 \%$ glycerol & D-IMK & ES & 22 & [5] \\
\hline
\end{tabular}

${ }^{a}$ media used: either Diago IMK (D-IMK) or f/2.

${ }^{\mathrm{b}}$ culture age: $\mathrm{EE}=$ early exponential (approximately 2 days after inoculation), ES = early stationary (8-10 days post inoculation), LS = late stationary (12-14 days post inoculation), LLS = late-late stationary (19-21 days post inoculation).

${ }^{c}$ the glycerol-induced signal (F585) strength relative to the autofluorescence signal (F587) in control cells (i.e., F585 was X times larger than F587).

ments, $R$. salina was grown in $\mathrm{f} / 2$ medium (Guillard 1975) in larger flasks (i.e., $150-250 \mathrm{ml}$ of rapidly growing $R$. salina was transferred to $1200 \mathrm{ml}$ in 2.51 flasks) and cells were collected in either early stationary or late stationary phases (i.e., 8-10 or 19 days, after inoculation, respectively). Most data presented in this report for $R$. salina involved cells grown in Daigo IMK that were harvested 2 days after inoculation; however, results from other media and/or duration of growth are also reported and are clearly indicated in Table 1.

\section{Steady state fluorescence assays}

For glycerol-induced fluorescence, $R$. salina cells were diluted to minimize/prevent re-absorption of fluorescence emissions [15]. Undiluted samples were scanned and preliminary fluorescence emission recorded; samples were diluted and scanned to ensure that signal strength was sufficiently strong for identification of peaks, but not off scale. Cultures with added glycerol were diluted with fresh medium to reach final glycerol concentrations of $0-80 \%$ $(\mathrm{v} / \mathrm{v})$. Steady-state room temperature fluorescence of $R$. salina was measured following Wyman [4] with some modifications. Fluorescence spectra were recorded on a Perkin Elmer model LS 55 Luminescence Spectrometer (Perkin Elmer Instruments, USA) with both emission and excitation slits of $5 \mathrm{~nm}$ and $100 \mathrm{~nm} / \mathrm{min}$ scan speeds. Samples were scanned 2-6 times, depending on the signal/noise ratios, and a single spectrum was generated for each sample based on a simple arithmetic average at each wavelength. Samples were separately excited with light at $440 \mathrm{~nm}$ ( Chl a), $470 \mathrm{~nm}$, $475 \mathrm{~nm}$ (Chl c), $490 \mathrm{~nm}$ (alloxanthin), or $520 \mathrm{~nm}$ (PE545); and emission spectra of the excited samples were recorded at individual wavelengths start- ing at $10 \mathrm{~nm}$ beyond the excitation light and extending to $750 \mathrm{~nm}$. A brief explanation of why we used $520 \mathrm{~nm}$ excitation is warranted. The $520 \mathrm{~nm}$ light is blue shifted relative to the major absorption band of PE545, but was utilized with high efficiency, and equally important are two additional factors for choosing $520 \mathrm{~nm}$ : (1) the fluorescence emissions of PE545, at F585 or F587, was minimized and (2) excessive leakage of the excitation beam into the emission detector was also minimized. Excitation spectra were recorded for specific emission signals; emissions at $685 \mathrm{~nm}$ (F685), $705 \mathrm{~nm}$ (F705) and $640 \mathrm{~nm}$ (F640) were proxies for PSII, PSI and PE545, respectively. The F645 signal is outside the absorption bands of PE545 but is within the range of reported fluorescence emissions for PE545 and is easily distinguished from signals arising from free Chl, LHCs (Light-Harvesting Complexes) and/or PSII.

\section{Steady state fluorescence spectrum, correction and deconvolution}

Fluorescence emission spectra were corrected for Photomultiplier Tube (PMT) sensitivity, and excitation spectra were deconvoluted into overlapping Gausian bands [16]. Briefly, the LS 55 instrument is shipped with a PMT with low sensitivity at long wavelengths; a red-sensitive PMT (e.g., R928, RCA4832) is recommended for analyses of glycerol-treated Synechococcus spp. [4] and cryptophytes [7]. Emission spectra were corrected by arithmetic division of $R$. salina spectra by correction factors, which allowed visualization of data as if a R928 red-sensitive PMT had been available. The long wavelength correction factors were generated based on the correction protocols of Perkin Elmer manufacturer. The printed spectra were manually 
digitized and used to calculate wavelength-specific correction factors over the range of interest (i.e., 530-750 nm). Excitation spectra of $R$. salina treated with glycerol were deconvoluted into overlapping Gaussian peaks using Excel Solver, an optimization Add-in for Excel; and spectra were not corrected for PMT sensitivity before analysis, as these qualitative results were marginally impacted by PMT sensitivity over the range of analysis (i.e., from $\sim 450$ to $\sim 600 \mathrm{~nm}$ ), as the standard PMT has good sensitivity in this wavelength range.

\section{Pulsed amplitude modulated (PAM) fluorescence assays}

Exponential phase cells of Rhodomonas sp. (R-FBT) were exposed to $0,10,20,40$, or $75 \%$ glycerol to determine the efficiency of phycobilisome decoupling by glycerol. Treated cells were incubated at $20^{\circ} \mathrm{C}$ for $5 \mathrm{~min}$ prior to examination at $525( \pm 5) \mathrm{nm}$ excitation and $685( \pm 5) \mathrm{nm}$ relaxation using BioTex Synergy HT plate reader (BioTek Instruments, Inc., USA). An additional experiment determined effects of $20 \%$ glycerol on Rhodomonas sp. (R-FBT). Photosynthetic ability (Chl $a$ fluorescence yield) was determined using a WALZ pulse amplitude modulation (PAM), and relaxing fluorescence was determined as previously described [17].

\section{Other procedures}

Cell densities were determined using a Neubauer Improved Brightline hemocytometer $\left(0.0025 \mathrm{~mm}^{2}\right)$. Triplicate $1.0 \mathrm{ml}$ aliquots were taken from each culture, and triplicate $10 \mu \mathrm{l}$ subsamples were counted from each aliquot.

\section{RESULTS AND DISCUSSION}

\section{Fluorescence emission spectroscopy}

Growth curves showed that $R$. salina reached the same cell density in $\mathrm{f} / 2$ as in Daigo IMK, but after a short lag period in $\mathrm{f} / 2$; PE545 was relatively easy to extract from cells grown in $f / 2$ medium, but was recalcitrant to extraction in Daigo IMK [5]. Daigo IMK has 2.6 times higher nitrate and contains ammonia and vitamins. We have not yet determined which of these factors (higher nitrate, addition of ammonia and/or addition of vitamins) is responsible for changes in PE545 extraction. Regardless, the following data are for $R$. salina grown in Daigo IMK (except as noted in Table 1).

The room temperature and steady state fluorescence emission of $R$. salina had low yield (Fig. 1a), as expected for cells in early exponential phase of growth. Excitation energy is transferred with high efficiency to PSI and/or PSII and used to drive ATP production and/or $\mathrm{CO}_{2}$ fixation. There were two conspicuous fluorescence emission peaks in the control cells (Fig. 1a): one at $685 \mathrm{~nm}$ (F685), previously associated with PSII [18]; and a larger peak at $587 \mathrm{~nm}$ (F587), previously associated with a pool of PE545 and not functionally connected to PSI or PSII $[19,20]$. This pool of PE545 is also referred to as the autofluorescent pool. There was a third, very small, peak at $702 \mathrm{~nm}$ (F702) likely associated with PSI. F702 in Rhodomonas spp. has very low signal strength at room temperature [18] which is difficult to detect. A fluorescence signal from Chl $a / c$ complexes was not observed, even when the excitation wavelength was at 440 or $465 \mathrm{~nm}$, the peak absorption bands for $\mathrm{Chl} a$ or $c$, respectively.

The room temperature and steady state fluorescence emission of $R$. salina was increased substantially following addition of glycerol, with the largest emission peak at $585 \mathrm{~nm}$ (F585), similar to the autofluorescence peak (F587) described above (Fig. 1b-g and Table 1). The increased fluorescence yield at F585 appeared within seconds after glycerol addition, an observation consistent with the PEassociated signal observed in Synechococcus spp. treated with 50\% glycerol [4]. The increased fluorescence signal was relatively stable for at least $1 \mathrm{~h}$ after glycerol addition (Fig. 1c-f), though the large increases were somewhat reduced in intensities with longer exposure times (i.e., longer than $1 \mathrm{~h}$, data not shown). Addition of glycerol had minimal effects on the $\mathrm{pH}$ of the growth medium, with only a small increase/decrease of signal. The effects of glycerol addition on the steady-state fluorescence yield of $R$. salina were verified in at least 12 independent experiments and are summarized in Table 1.

Glycerol concentrations of 20 to $30 \%$ routinely and reproducibly resulted in the greatest increase of F585 signal in R. salina (Fig. 1cd) at 15-34 times compared with untreated cells depending upon culture conditions (e.g., media used and/or age of culture post inoculation; Table 1). F585 was also increased in 40 and 50\% glycerol (Fig. 1ef) but less so than at $20-30 \%$ glycerol. At very high glycerol concentrations (i.e., 80\%), F585 was of low intensity and shifted 5-7 nm toward the blue (to F579, Fig. 1g); similar shifts were observed previously in purified PE545 and ascribed to denaturation of pigments and/or disruption of pigmentpigment and/or pigment-protein interactions within PE545 [5, 7]. At optimal concentrations of glycerol (i.e., 20 or $30 \%$ ), F585 was stable for at least $1 \mathrm{~h}$ following addition of glycerol (Fig. 1cd). 


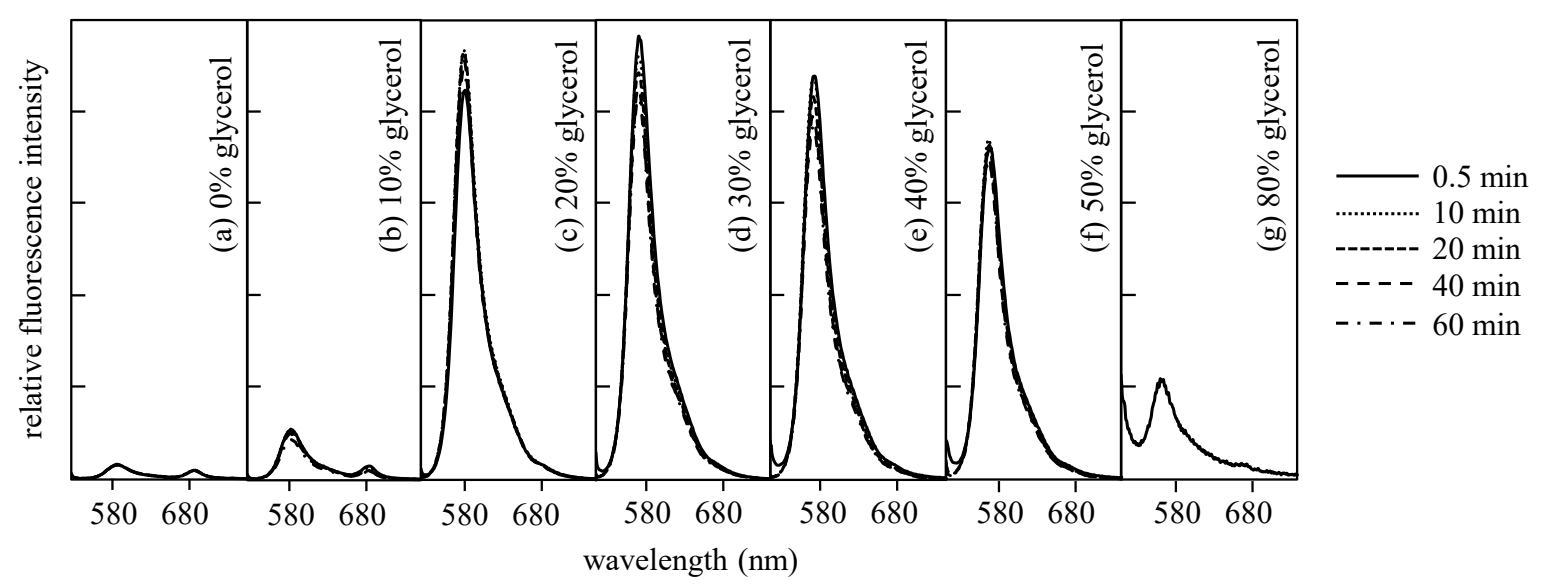

Fig. 1 In vivo steady state fluorescence emissions arising from $520 \mathrm{~nm}$ excitation of $R$. salina treated with different concentrations of glycerol $(0-80 \%, a-g)$ at various periods of time $(0.5-60 \mathrm{~min})$. A time-series for the $80 \%$ glycerol sample was not conducted, as the signal was initially low and did not change appreciably over $1 \mathrm{~h}$.

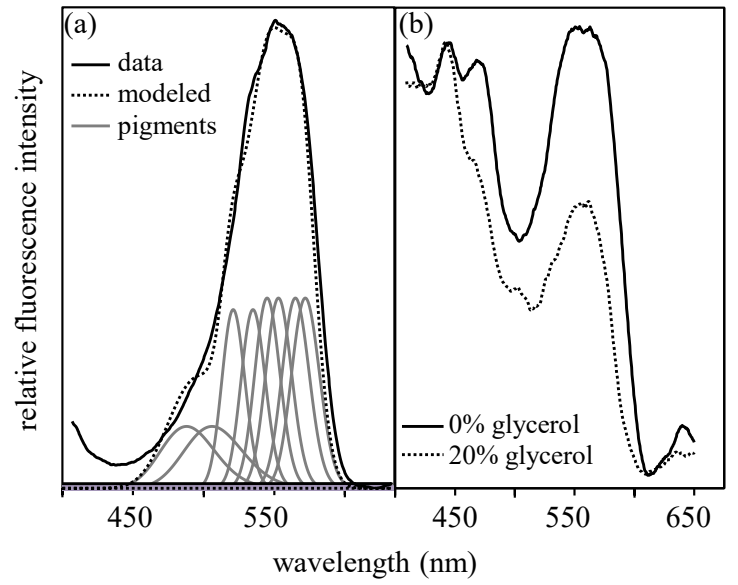

Fig. 2 In vivo excitation spectra of R. salina. In (a), the data for cells in $20 \%$ glycerol with fluorescence emission at $645 \mathrm{~nm}$ were plotted with the modeled data derived by curve deconvolution based on the eight known pigments of PE545 at the absorption maxima of 488, 506, 521, $535,545,553,565$, and $572 \mathrm{~nm}$, respectively. In (b), the two spectra for cells in either $0 \%$ or $20 \%$ glycerol with fluorescence emission at $685 \mathrm{~nm}$ were normalized to their respective Chl $a$ excitation maxima at $\sim 440 \mathrm{~nm}$.

\section{Fluorescence excitation spectroscopy}

The fluorescence emission of glycerol-treated $R$. salina peaked at $\mathrm{F} 585$, but the spectrum had a very broad line shape, extending well beyond F585 into longer wavelengths (Fig. 1c-f). These red emission signals could arise from Chl-protein complexes (e.g., PSI, PSII, or $\mathrm{Chl} a / c$ ) or from
PE545, which is known to have a 'red tail' of fluorescence. To distinguish these possible origins of the fluorescence emission in glycerol-treated cells, excitation spectra were generated for a number of wavelengths (i.e., every $5 \mathrm{~nm}$ from 585 $710 \mathrm{~nm}$ ). The F645 signal was chosen for detailed presentation (Fig. 2a) because this wavelength was considerably longer than the primary F585 peak but was near a long wavelength fluorescence shoulder of PE545 [20] associated with two MBV pigments on the $\alpha_{1}$ and $\alpha_{2}$ subunits [18]. Moreover, it was considerably blue shifted relative to emission peaks expected for Chl $a / c(683 \mathrm{~nm})$, PSII $(685 \mathrm{~nm})$, or PSI $(702 \mathrm{~nm})$. In other words, the F645 signal was easily ascribed to PE545, and it could be readily distinguished from Chl-protein signals.

The in vivo excitation spectrum of F645 in cells treated with $20 \%$ glycerol had a major peak at $545 \mathrm{~nm}$ with a shoulder at $568 \mathrm{~nm}$ (Fig. 2a). The shape of the excitation spectrum was similar to the absorption spectrum of isolated PE545 $[5,20]$ and was well modeled by eight bands, one for each of the eight pigments in the PE545 heterodimer (Fig. 2a). The two excitation bands on the blue edge (i.e., 488 and $506 \mathrm{~nm}$ ) had less intense signals and broadened absorption features in glycerol-treated cells, similar to those in isolated PE545 [20]. However, the presence of an absorption band at $488 \mathrm{~nm}$ is not expected for PEB absorption, but it has been reported previously in $R$. salina [21].

The following data were generated to answer a straight-forward question: was the increase of fluorescence induced by glycerol (Fig. 1) related solely 
to PE545, or did Chl-protein complexes contribute to the signal? In brief, the excitation spectrum for F645 (Fig. 2a) provided evidence that the fluorescence 'red tail' was derived from PE545 but did not rule out contributions from Chl-protein complexes, especially at wavelengths longer than $645 \mathrm{~nm}$. It is important to point out that it would not be surprising to have $\mathrm{F}$ signals from Chl-protein complexes in these spectra, as they were generated using 'whole cells' with a full complement of Chl $a / c$, PSII, and PSI; and no steps had been taken to separate these Chl-protein complexes before fluorescence assays were performed. For instance, PE545 could be physically and energetically coupled to Chl $a / c$ and/or PSII after glycerol treatment; and this would give an excitation spectrum with a large PE545 component, though the emission would be from one (or both) of the Chl-protein complexes with high fluorescence when uncoupled from the electron transport chain. To determine the extent, if any, that Chl $a / c$ and/or PSII contributed to the fluorescence 'red tail', excitation spectra for F685 nm in glyceroltreated $R$. salina was examined and compared to the excitation spectra for F685 in cells without glycerol (Fig. 2b). In the control cells (without glycerol), F685 excitation energy was derived from pigments with absorption bands corresponding to Chl $a(440 \mathrm{~nm})$, Chl c (465 nm), and PE545 (545 nm peak with a shoulder). The same absorption bands, with peaks at 440, 465, and $545 \mathrm{~nm}$, were present in glycerol-treated cells; but the relative strengths of these signals were very different from those in the control cells. The contributions from both Chl $c$ and PE545 were greatly reduced compared with the contribution from Chl $a$ following addition of glycerol (Fig. 2b). The most straightforward explanation for these results was that F685 excitation comes from three potential sources in the control cells: (1) the two core antenna CP685 and CP695 of PSII, (2) Chl $a / c$ complexes, and (3) PE545; all three of which were energetically coupled in the control cells. In contrast, F685 excitation in glycerol-treated cells arose from only two sources, a relatively small F signal from the core antenna of PSII (i.e., CP685 and CP695) and a larger F signal from the 'red tail' of PE545, a light-harvesting complex that was no longer energetically coupled to PSII. Stated differently, PE545 was energetically coupled to PSII in the control cells resulting in fluorescence emission at $685 \mathrm{~nm}$, while PE545 in the glycerol-treated cells was no longer coupled to PSII and, as a result, gave rise to a F585 signal that extended to, and overlapped with, PSII at F685.
The excitation spectrum of F702 (data not shown), associated with PSI in the control cells, was qualitatively similar to the excitation spectrum of F685 (Fig. 2b); but the extremely low fluorescence yield of PSI in $R$. salina and the low sensitivity of the PMT used in this study at $702 \mathrm{~nm}$ made it difficult to draw definitive conclusions, as the signal/noise ratio in the $400-450 \mathrm{~nm}$ range complicated the data analysis. Despite these limitations, the F702 excitation spectrum in cells treated with $20 \%$ glycerol appeared to be similar to the excitation spectrum of F685, with a major contribution from Chl $a$ and a larger signal associated with the 'red tail' of uncoupled PE545 (data not shown).

The fluorescence excitation spectroscopy data in Fig. 2 provided strong evidence that the glycerolmediated increase in the fluorescence signal of $R$. salina was overwhelmingly from pigments whose absorption properties mirrored those of PE545 when fluorescence was excited at $520 \mathrm{~nm}$ and emission measured at $585 \mathrm{~nm}$ (Fig. 2a; and other wavelengths in the 550-600 nm range, data not shown). Moreover, these data provided evidence, but not a proof, that the long 'red tail' of fluorescence observed in glycerol-treated $R$. salina arose from PE545. Although a 'red tail' fluorescence emission signal can be seen in spectra of isolated PE545, it was not clear if this signal would be preserved in the presence of glycerol; and the following work addressed this issue.

\section{Fluorescence analysis of supernatants and filtrates of $R$. salina treated with glycerol}

The effect of glycerol, if any, on the 'red tail' of PE545 was investigated with three complementary approaches. In the first approach, we used light microscopy and epifluorescence microscopy of glycerol-treated $R$. salina to show that glycerol addition resulted in cell rupture and release of intracellular constituents into the growth medium; while the extracellular pool contained very small particles with fluorescence properties qualitatively similar to those of the control cells (i.e., cells not treated with glycerol). The glycerol induced rupture of $R$. salina was not expected; we expected to see cells shrink. We explained this rupture based on glycerol acting as tonicity [22].

In the second approach, it was hypothesized that a portion of the PE545 pool had been released into the extracellular pool and that the F585 signal would remain in suspension following low speed centrifugation $(13000 \times g$ for $5 \mathrm{~min}$ ) of the glyceroltreated cells, which should sediment whole cells, 


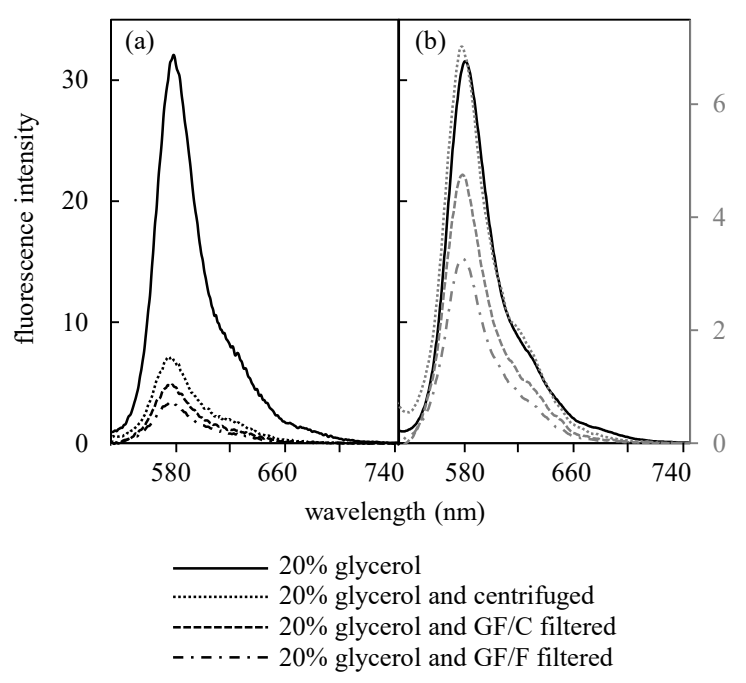

Fig. 3 Steady state fluorescence emissions arising from $520 \mathrm{~nm}$ excitation of R. salina. Cells treated with $20 \%$ glycerol (control) were either centrifuged at $13000 \times g$ for $5 \mathrm{~min}$ before analysis of the supernatant or filtered through either GF/C or GF/F before analysis of the filtrates. The four emission spectra in (a) were plotted on the same scale (left axis) as recorded; in (b), spectra for the control and the centrifugation supernatant were adjusted to the same peak heights and the filtrates plotted on the same scale (right axis) as the supernatant.

but not pigment-protein complexes; and this hypothesis was proved accurate (Fig. 3). The F585 signal in the supernatant of glycerol-treated cells was reduced in intensity following centrifugation by about $70-80 \%$, indicating that about $20-30 \%$ of the pigments responsible for F585/87 remained in the supernatant. Qualitatively, the spectral properties of the supernatants of glycerol-treated cells were largely unchanged following centrifugation, especially as related to the F585/87 signals (Fig. 3ab); and signals associated with PSII and/or PSI were still detectable in the supernatant, but with greatly diminished intensity suggesting that they were mostly localized intracellularly (data not shown).

The third approach was based on the observation that a portion of the F585 signal remained in the supernatant following low-speed centrifugation (Fig. 3), therefore it is possible that F585 pigments also passed through filter pads. As a background, a dissolved material in seawater is generally defined as matter that passes through a given filter (e.g., 0.22 or $0.45 \mu \mathrm{m}$ pore size), whereas a particulate material is retained on the filter pad. Glycerol- treated cells were subjected to filtration using three different filter pads, with pore sizes of $0.22,0.45$, or $0.7 \mu \mathrm{m}$. Results indicated that approximately $4-16 \%$ of $F 585$ passed through the filter pads in a pore-size-dependent fashion (Fig. 3a). Looking at the material that passed through the GF/C and $\mathrm{GF} / \mathrm{F}$ filters in more details, the emission peaks were centered at $585 \mathrm{~nm}$, identical to F585 signals before filtration; and the filtrate emission spectra also exhibited pronounced 'red tail' that extended to $750 \mathrm{~nm}$ (Fig. 3ab). The excitation spectra of the two filtrates were devoid of Chl $a$ and Chl $c$ contributions (i.e., absorption at $440 \mathrm{~nm}$ and $465 \mathrm{~nm}$, respectively; data not shown).

\section{PAM analysis of Rhodomonas sp. treated with glycerol}

The effects of glycerol were assessed on the second strain of Rhodomonas (R-FBT) using Pulse Amplitude Modulated (PAM) fluorescence. PAM fluorescence provides a non-invasive means of measuring photosynthetic electron flow (ETR), particularly the electron flow around PSII reaction center [23]. Initial experiments showed that $10 \%$ glycerol minimally impacted ETR and yield, while glycerol concentrations of $20 \%$ or greater had dramatic effects that were independent of glycerol concentrations (data not shown). Data for $20 \%$ glycerol are detailed with both ETR (Fig. 4a) and yield (Fig. 4b) followed the expected light response curves, with increased ETR and reduced yield as light intensity (PAR) increased. In contrast, ETR was reduced to levels that were near instrument detection limits in glycerol-treated cells (Fig. 4a) but did appear to exhibit PAR dependent increases at the lower light intensities tested (Fig. 4a). For the ETR values of regular cells which kept rising in the control sample (Fig. 4a), it can occur and be related to microalgal strain, cultivation conditions, physiological status, and microalgal growth [24]. Yield estimates with glycerol-treated cells are informative but likely unreliable because of the low ETR values recorded under this condition (Fig. 4b). Overall, the PAM data clearly indicates in the second strain of Rhodomonas (R-FBT) that $20 \%$ glycerol had substantial impacts on photochemistry. Similar results were obtained in preliminary experiments with $R$. salina using an AquaPen-C device (data not shown). The PAM data, combined with photomicrographs (data not shown) and filtration data (Fig. 3), strengthen the hypothesis that glycerol effects extended beyond uncoupling PE545 from its role as a light-harvesting pigment and extended to other photochemical processes. 

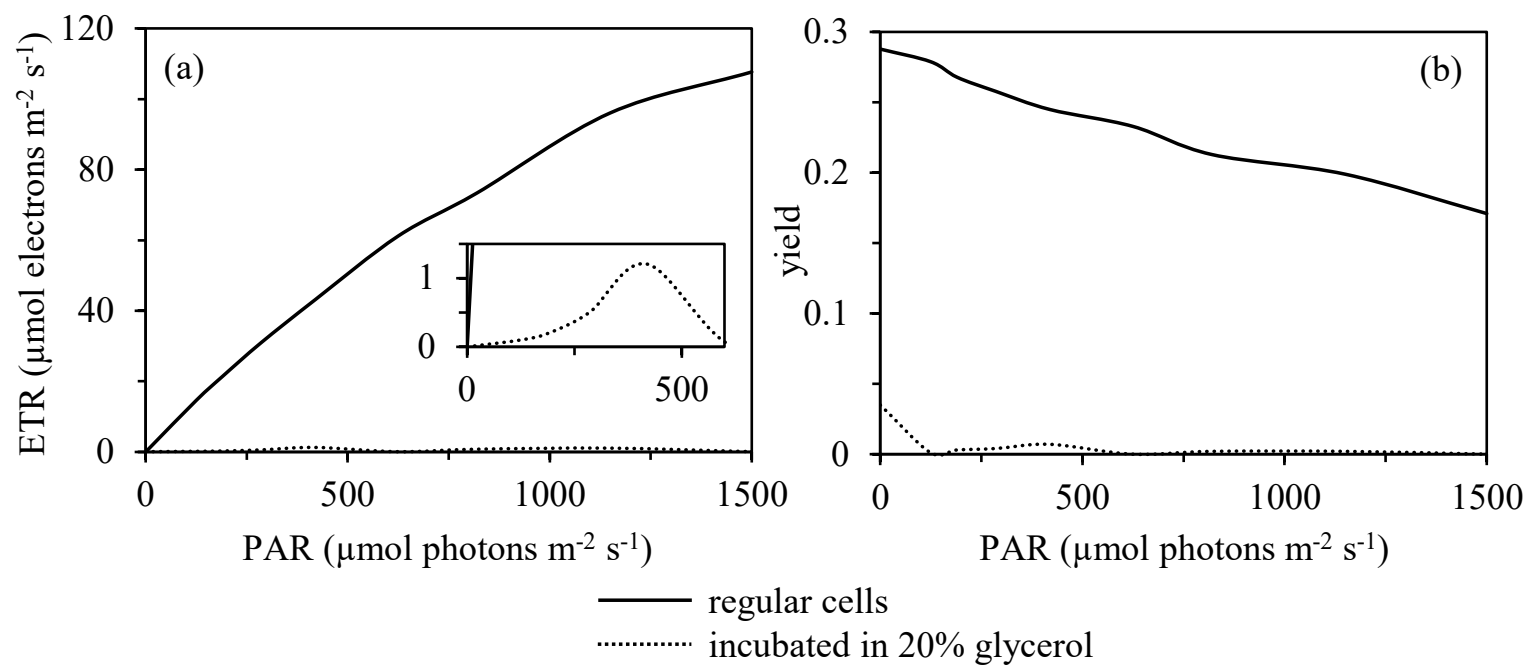

Fig. 4 ETR (a) and yield (b) of the control and the 20\% glycerol-treated Rhodomonas (R-FBT strain) measured with PAM.

Non-photochemical Quenching (NPQ, Fig. S1) measured in R. salina with and without addition of $20 \%$ glycerol also supported the PAM data.

\section{CONCLUSION}

Rhodomonas spp. is an abundant and important prey source ecologically. This study describes a simple procedure, based on glycerol-induced increases in fluorescence emission of PE545, for rapid analyses of phycobilins in Rhodomonas spp. 20-30\% glycerol was optimal for short-term experiments, while higher concentrations or prolonged exposure to glycerol could be deleterious to PE545 stability. For field work, results shown here with Rhodomonas spp. strongly suggest that size-fractionated filtration should be included as part of the procedure to allow clear separation of large cells, such as Rhodomonas spp., from small cells, such as Synechococcus spp., while simultaneously eliminating other cells that potentially could confound interpretation of results. The inclusion of size fractionation would seem especially important in coastal waters and/or waters collected near the bottom of the euphotic zone, areas likely to have abundant cryptophytes. One potentially fertile area for future research lies in evaluation of increases in fluorescence induced by glycerol with other pigmented cryptophytes. In other words, is Rhodomonas spp. unique, or do other cryptophytes share this trait?

\section{Appendix A. Supplementary data}

Supplementary data associated with this article can be found at http://dx.doi.org/10.2306/ scienceasia1513-1874.2021.062.

Acknowledgements: The study was financially supported by the 90th Anniversary of Chulalongkorn University Scholarship, Grants for Development of New Faculty Staff (Ratchadaphiseksomphot Endowment Fund) and Visiting Professorship (Kanchanaphisek Chalermphrakiat Endowment Fund). Our sincere thanks are given to Assoc. Prof. Dr. Thaithaworn Lirdwitayaprasit and Ingon Thongcomdee (Algal Culture Laboratory, Department of Marine Science, CU) who provided help with algal cultures.

\section{REFERENCES}

1. Zimba PV (2012) An improved phycobilin extraction method. Harmful Algae 17, 35-39.

2. Lawrenz E, Fedewa EJ, Richardson TL (2011) Extraction protocols for the quantification of phycobilins in aqueous phytoplankton extracts. J Appl Phycol 23, 865-871.

3. Wyman M, Gregory R, Carr N (1985) Novel role for phycoerythrin in a marine cyanobacterium, Synechococcus strain DC2. Science 230, 818-820.

4. Wyman M (1992) An in vivo method for the estimation of phycoerythrin concentrations in marine cyanobacteria (Synechococcus spp.). Limnol Oceanogr 37, 1300-1306.

5. Karnjanapak C (2016) Evaluation of phycobilins in marine cryptomonad Rhodomonas salina and marine cyanobacteria Synechococcus spp. Master thesis, Chulalongkorn Univ, Bangkok, Thailand. 
6. Chaloub RM, Motta NMS, de Araujo SP, de Aguiar PF, da Silva AF (2015) Combined effects of irradiance, temperature and nitrate concentration on phycoerythrin content in the microalga Rhodomonas sp. (Cryptophyceae). Algal Res 8, 89-94.

7. MacColl R, Malak H, Gryczynski I, Eisele LE, Mizejewski GJ, Franklin E, Hesham S, Montellese D, et al (1998) Phycoerythrin 545: monomers, energy migration, bilin topography, and monomer/dimer equilibrium. Biochemistry 37, 417-423.

8. Johnson MD, Beaudoin DJ, Frada MJ, Brownlee EF, Stoecker DK (2018) High grazing rates on cryptophyte algae in Chesapeake Bay. Front Mar Sci 5, 1-13.

9. Gieskes WWC, Kraay GW (1983) Dominance of cryptophyceae during the phytoplankton spring bloom in the central North Sea detected by HPLC analysis of pigments. Mar Biol 75, 179-185.

10. Wollschläger J, Wiltshire KH, Petersen W, Metfies K (2015) Analysis of phytoplankton distribution and community structure in the German Bight with respect to the different size classes. J Sea Res 99, 83-96.

11. Dubelaar GBJ, Geerders PJF, Jonker RR (2004) High frequency monitoring reveals phytoplankton dynamics. J Environ Monit 6, 946-952.

12. Lokstein H, Steglich C, Hess WR (1999) Lightharvesting antenna function of phycoerythrin in Prochlorococcus marinus. Biochim Biophys Acta 1410, 97-98.

13. Williams WP, Saito K, Furtado D (1981) Use of lateral phase separations as a probe of photosynthetic membrane organization. In: Akoyunoglou G (ed) Photosynthesis III. Structure and Molecular Organisation of the Photosynthetic Apparatus, Balapan International Science Services, Philadelphia, pp 97-106.

14. Heathcote P, Wyman M, Carr NG, Beddard GS (1992) Partial uncoupling of energy transfer from phycoerythrin in the marine cyanobacterium Synechococcus sp. WH7803. Biochim Biophys Acta Bioenerg 1099, 267-270.

15. Mohanty P, Braun BZ, Govindjee (1972) Fluorescence and delayed light emission in Tris-washed chloroplasts. FEBS Lett 20, 273-276.

16. Garlaschi FM, Zucchelli G, Giavazzi P, Jennings
RC (1994) Gaussian band analysis of absorption, fluorescence and photobleaching difference spectra of D1/D2/cyt b-559 complex. Photosynth Res 41, 465-473.

17. Schreiber U (2004) Pulse-amplitude-modulation (PAM) fluorometry and saturation pulse method: an overview. In: Papageorgiou GC, Govindjee (eds) Chlorophyll a Fluorescence, A Signature of Photosynthesis, Springer, The Netherlands, pp 279-319.

18. Doust AB, Wilk KE, Curmi PMG, Scholes GD (2006) The photophysics of cryptophyte light-harvesting. $J$ Photochem Photobio A 184, 1-17.

19. van der Weij-De Wit $C D$, Doust $A B$, van Stokkum IHM, Dekker JP, Wilk KE, Curmi PMG, Scholes GD, van Grondelle R (2006) How energy funnels from the phycoerythrin antenna complex to photosystem I and photosystem II in cryptophyte Rhodomonas CS24 cells. J Phys Chem B 110, 25066-25073.

20. Doust AB, Marai CN, Harrop SJ, Wilk KE, Curmi PM, Scholes GD (2004) Developing a structure-function model for the cryptophyte phycoerythrin 545 using ultrahigh resolution crystallography and ultrafast laser spectroscopy. J Mol Biol 344, 135-153.

21. Kana R, Prašil O, Mullineaux CW (2009) Immobility of phycobilins in the thylakoid lumen of a cryptophyte suggests that protein diffusion in the lumen is very restricted. FEBS Lett 583, 670-674.

22. Silverthorn DU (2011) Osmolarity and tonicity: an inquiry laboratory using plant material. In: Tested Studies for Laboratory Teaching, Proceedings of the Association for Biology Laboratory Education, 32, pp 135-150.

23. Schreiber U, Schliwa U, Bilger W (1986) Continuous recording of photochemical and non-photochemical chlorophyll fluorescence quenching with a new type of modulation fluorometer. Photosynth Res 10, 51-62.

24. Malapascua JRF, Jerez CG, Sergejevová M, Figueroa FL, Masojídek J (2014) Photosynthesis monitoring to optimize growth of microalgal mass cultures: application of chlorophyll fluorescence techniques. Aquat Biol 22, 123-140. 


\section{Appendix A. Supplementary data}
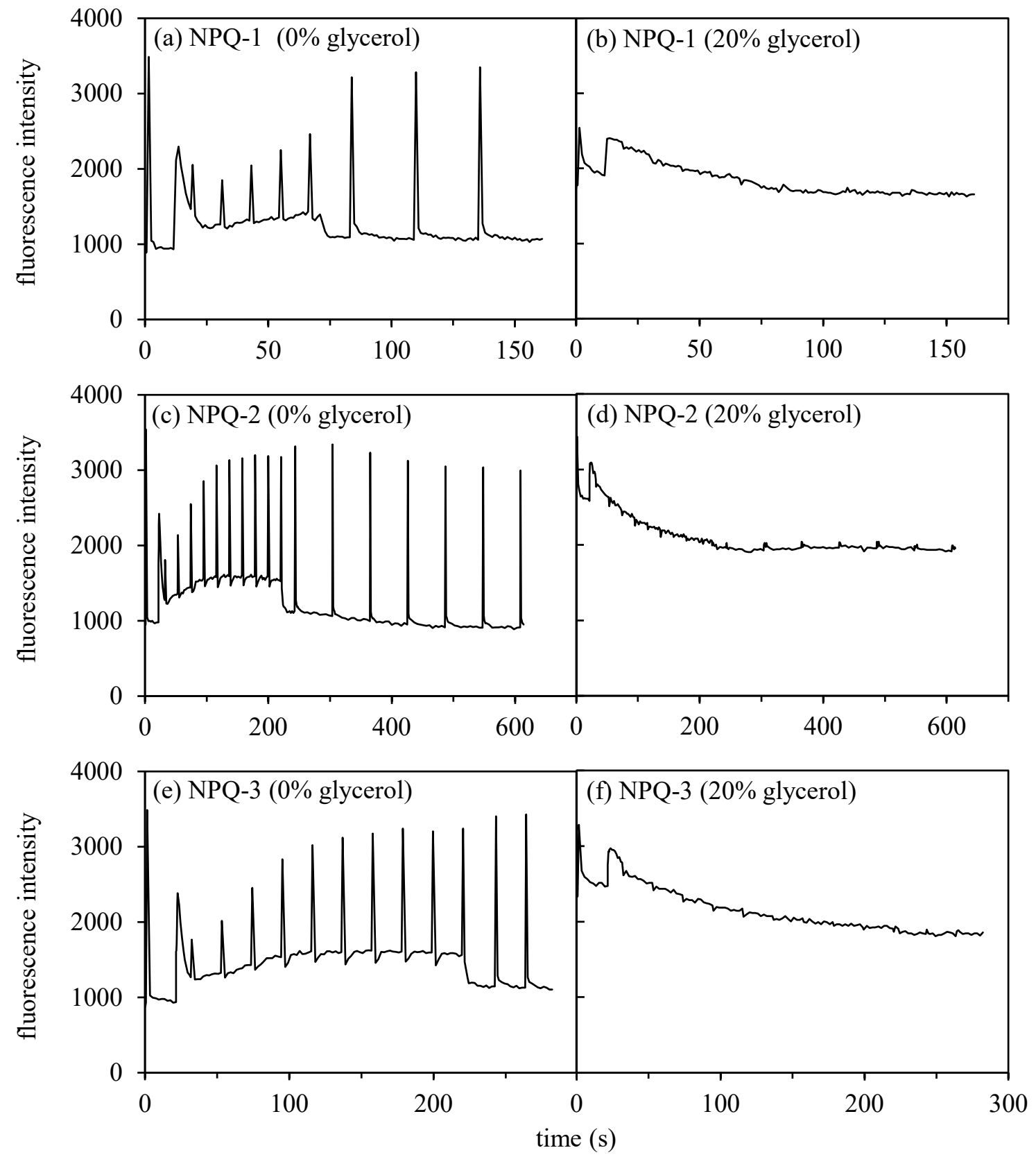

Fig. S1 Non-photochemical quenching (NPQ) of the controls (a, c and e) and the $20 \%$ glycerol-treated $R$. salina at day-2 culture (b, d and f), induced during illumination with 450-nm light and measured with AquaPen-C under three predefined profiles (NPQ-1, NPQ-2 and NPQ-3). 